\title{
Adenocarcinoma lung presenting with meningeal carcinomatosis
}

\author{
Ramakant Dixit ${ }^{1}$, Mukesh Goyal' ${ }^{1}$, Paras Nuwal' ${ }^{2}$, Varna Indushekhar ${ }^{2}$ \\ ${ }^{1}$ Department of Respiratory Medicine; ${ }^{2}$ Department of Pathology, Jawaharlal Nehru Medical College, Ajmer, India
}

\begin{abstract}
Meningeal infiltration by malignant metastasis process without brain parenchymal involvement is very unusual event in patients with bronchogenic carcinoma. This manuscript describes a case of adenocarcinoma right lung in a 48-year old male having persistent headache. The cerebrospinal fluid cytology revealed the presence of metastatic deposits of adenocarcinoma lung that confirmed the diagnosis of meningeal carcinomatosis.
\end{abstract}

\section{Introduction}

Meningeal carcinomatosis represents dissemination of malignant cells to the leptomeninges and subarachnoid space

Correspondence: Dr. Ramakant Dixit, Senior Professor \& Head, Department of Respiratory Medicine, Jawaharlal Nehru Medical Medical College, Ajmer 305001, Rajasthan, India.

E-mail: dr.ramakantdixit@gmail.com

Key words: adenocarcinoma lung; meningeal carcinomatosis; headache.

Contributions: RD, study concept and design, data acquisition, analysis and interpretation, manuscript drafting and critical revision for important intellectual content; MG, study concept and design, manuscript drafting and critical revision for important intellectual content; PN, VI, study concept and design. All the authors have read and approved the final version of the manuscript and agreed to be accountable for all aspects of the work.

Conflict of interest: The authors declare that they have no competing interests, and all authors confirm accuracy.

Ethics approval: No ethical committee approval was required for this case report by the Department, because this article does not contain any studies with human participants or animals.

Received for publication: 2 June 2020 .

Accepted for publication: 13 September 2021.

${ }^{\circ}$ Copyright: the Author(s), 2021

Licensee PAGEPress, Italy

Monaldi Archives for Chest Disease 2022; 92:1427

doi: 10.4081/monaldi.2021.1427

This article is distributed under the terms of the Creative Commons Attribution Noncommercial License (by-nc 4.0) which permits any noncommercial use, distribution, and reproduction in any medium, provided the original author(s) and source are credited. that mostly represent a late complication of long standing neoplastic disease. This process can occur in both non-central nervous system (CNS) tumours as well as CNS tumours. The diagnosis is difficult when meningeal dissemination occurs without brain parenchymal involvement which represent its pure form and also this is first ever manifestation of otherwise hidden tumour [1]. The present report describes such a case where adenocarcinoma lung presented with meningeal carcinomatosis.

\section{Case Report}

A 48-year old male presented with complaints of cough without expectoration, right sided chest pain for three months and an episode of scanty haemoptysis three days back. He also had persistent headache over last one month. Headache was at frontal-parietal region, more in morning and progressively increasing. He denied any history of fever, breathlessness, running nose, joint pain, vomiting, visual disturbance, giddiness, limb weakness etc. He was current smoker and farmer by occupation. His past medical history and family history was unremarkable.

On physical examination, he was average built, nourished, well oriented and without anaemia, clubbing, cyanosis, palpable lymphadenopathy or oedema etc. Vital parameters were all normal. Respiratory examination revealed nothing abnormal except dull percussion note and slight decreased intensity of breath sounds at right lower axillary area. There was no tenderness over paranasal sinus areas. On central nervous system examination, the higher mental functions, motor system, sensory system, cranial nerve examination, etc. were normal, however; there was terminal neck rigidity. The Romberg's test was also normal. His ocular examination and other system examination were absolutely normal.

On investigations, blood counts and biochemistry was within normal limit. His ECG was normal with $\mathrm{SpO}_{2}$ of $97 \%$ at room air. His sputum Cartridge Based Nucleic Acid Amplification Test (CBNAAT) for Mycobacterium tuberculosis and HIV serology testing were negative. X-ray chest PA view revealed a lobulated mass at right lower zone above diaphragm that was confirmed on CT scan chest (Figure $1 \mathrm{a}, \mathrm{b}$ ). A CT guided FNAC of peripheral pulmonary mass revealed haemorrhagic aspirate with clumps of atypical cells in singles, clusters and at places in acinar pattern having irregular, hyperchromatic nuclei with prominent nucleoli and abundant eosinophilic cytoplasm (Figure 2). The cytological features were consistent with the diagnosis of adenocarcinoma lung. Molecular studies for epidermal growth factor receptor (EGFR)-mutation and anaplastic lymphoma kinase (ALK)-positive cells could not be done due to non availability. X-ray paranasal sinus, skull, cervical and dorsal spine were all normal. CT scan head was absolutely normal. MRI brain revealed focal 
meningeal enhancement with no space occupying lesion, brain parenchymal or ventricular abnormality. On lumbar puncture, CSF was clear but pressure was slightly raised. CSF examination revealed protein $64 \mathrm{mg} / \mathrm{dl}$, glucose $42 \mathrm{mg} / \mathrm{dl}$, ADA $02 \mathrm{IU}$, cells $67 / \mathrm{mm} 3$ with almost all lymphocytes. On careful examination of CSF cytology, few abnormal atypical cells were also seen. The cyto-morphological features of these cells were same as with FNAC of lung lesion (Figure 3). The CSF was sterile for bacterial culture and negative for $M$. tuberculosis on CBNAAT. The patient was finally diagnosed as stage 4 non-small cell lung carcinoma (NSCLC) and referred for further management by medical oncologist.

\section{Discussion}

The meningeal carcinomatosis, also called neoplastic meningitis or leptomeningeal carcinomatosis is diffuse infiltration of leptomeninges and subarachnoid space by the metastatic carcinomas. Although, it occurs in 3-8\% of all cancer patients, this complication is relatively more common in haematolymphoid malignancies i.e. lymphomas and leukaemias; compared to solid tumours that include breast, lung, stomach, ovary, gall bladder, melanoma, etc. [2]. Among lung cancers, it is more common in small cell carcinoma and it may be the presenting manifestation in non-small cell

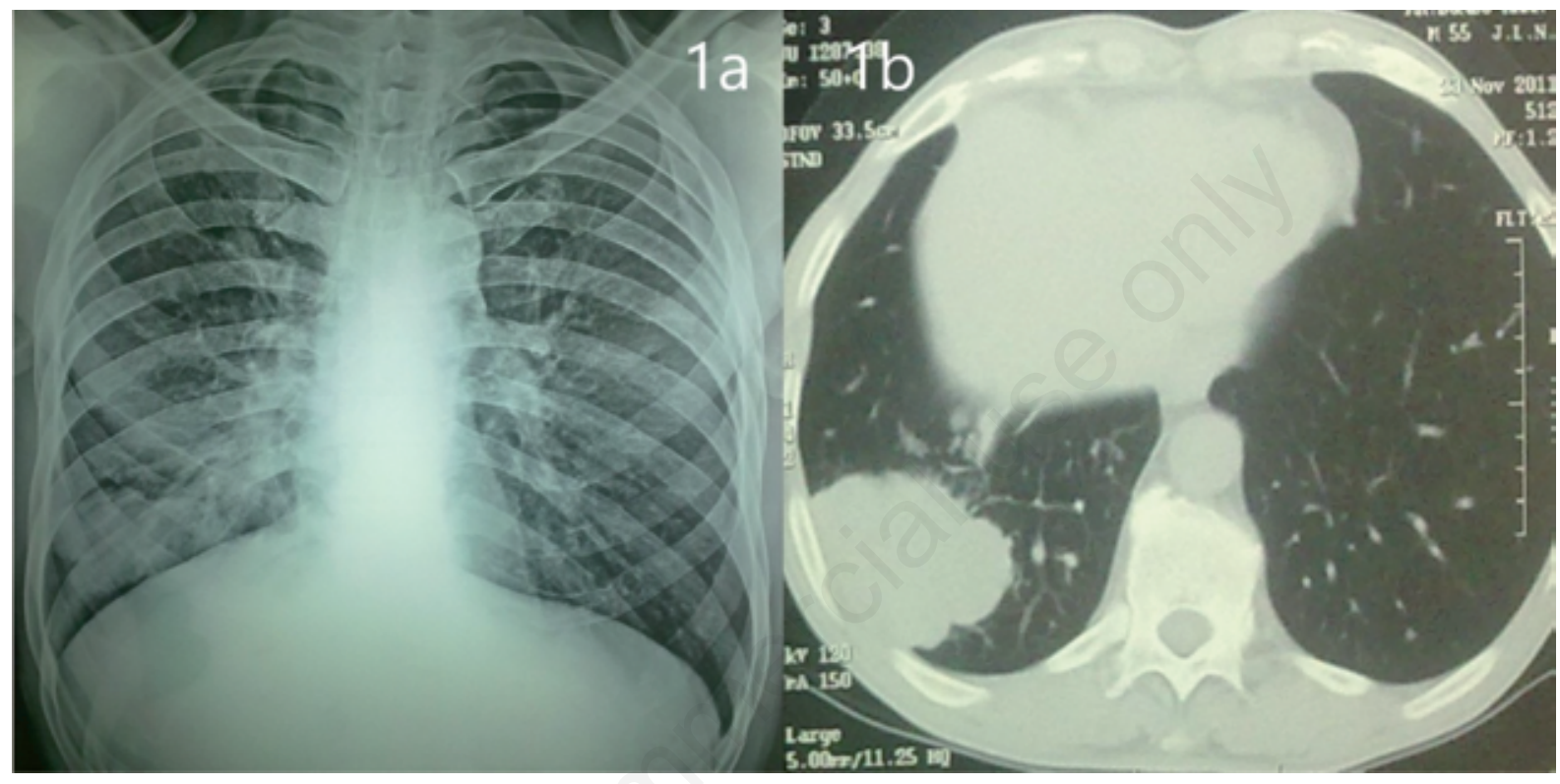

Figure 1. X-ray chest (a) and CT image (b) showing a lobulated lung mass at right lower lobe.

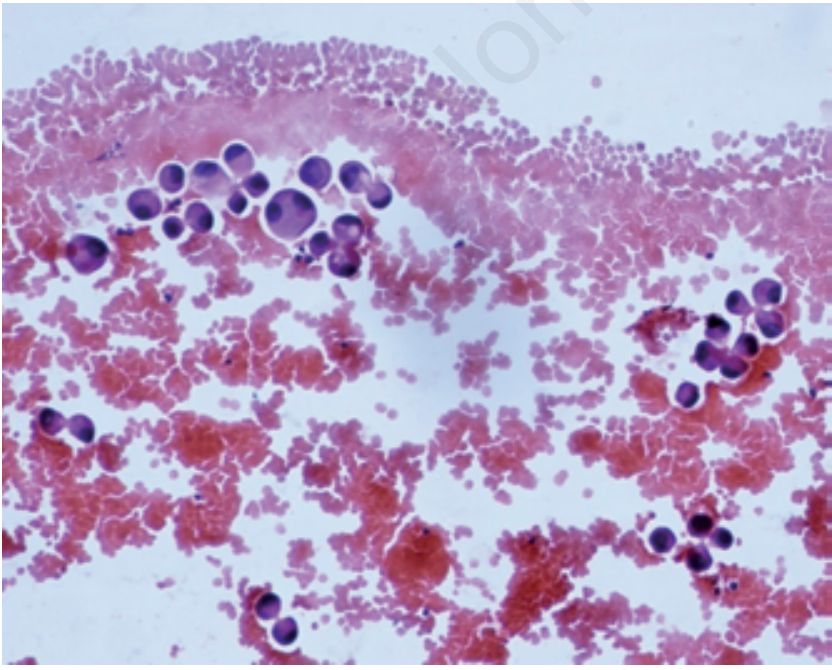

Figure 2. Photomicrograph of FNAC lung lesion showing round to oval cells in singles, clusters, few are 'binucleate' with hyperchromatic nuclei and vacuolated cytoplasm in RBCs rich background (H\&Ex200).

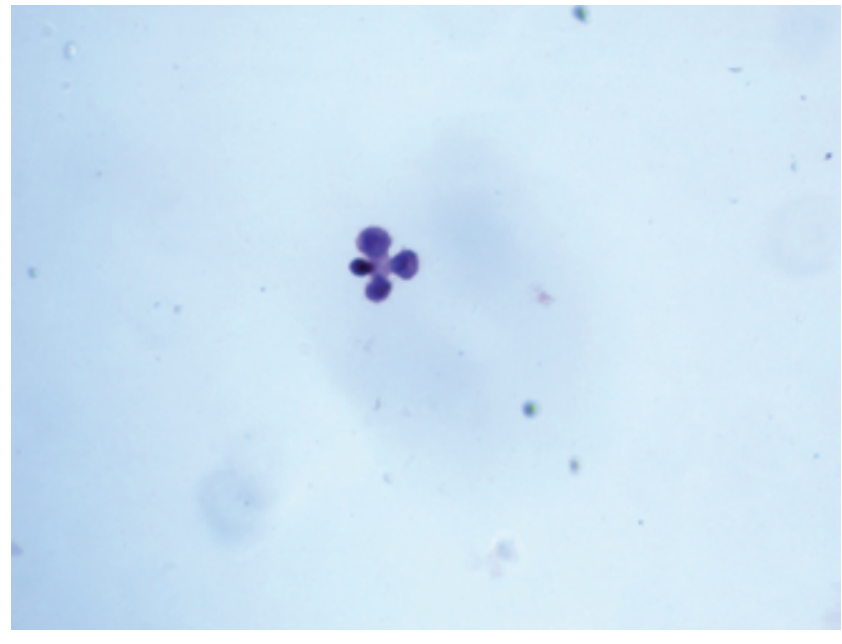

Figure 3. CSF cytology showing scanty cellular smear with round to oval cells having irregular hyperchromatic nuclei, moderate eosinophilic cytoplasm against a clear background (H\&Ex200). The cytological morphology and pattern of cells is same as in Figure 2. 
lung carcinoma [3]. Among NSCLC, adenocarcinoma is the most common histological cell type to cause leptomeningeal metastasis especially those with EGFR mutation and ALK positive stains [4].

A thorough neurological history and examination is must to suspect meningeal carcinomatosis. The signs and symptoms may be cerebral (headache, altered mental status, vomiting, unconsciousness, seizures, cognitive dysfunction etc.); cranial (visual defects, hearing disturbance, dysphagia etc.); spinal (facial weakness, sensory loss, back pain, bowel and bladder dysfunction etc.) and nuchal rigidity. Initially the clinical signs and symptoms may be subtle, difficult to elicit and might get easily overlooked [5].

The diagnosis is confirmed on CSF cytology or leptomeningeal biopsy (gold standard). The CSF cytology is simple, reliable and accurate, with least false positivity. The diagnostic yield of cytology increases on repeat examinations of CSF. The CSF analysis usually shows raised protein, pleocytosis, and hypoglycorrhachia. The detection of tumour markers in CSF may be useful in cytologically negative cases, however; its clinical applicability is limited. CT scan brain may be normal in majority of patients. Enhanced MRI brain is more informative and sensitive in demonstrating abnormalities than CT scan. Contrast T1-weighted and FLAIR sequences MRI studies are more sensitive to detect meningeal carcinomatosis [6].

Median survival is 4-6 weeks in untreated patients and 2-3 months among those who get treatment [7]. According to the EANO-ESMO clinical practice guidelines, the aim of treatment of leptomeningeal metastasis is to prolong survival with an acceptable quality of life, and to prevent or delay the neurological deterioration. The treatment options include radiotherapy, intrathecal chemotherapy, systemic chemotherapy and surgical intervention such as ventricular peritoneal shunts. A platinum based-chemotherapy (including pemetrexed, gemcitabine, paclitaxel for NSCLC) can be chosen for treating meningeal metastasis. Subset of patients having EGFR mutation may be benefit from first generation (gefitinib, erlotinib) and second generation (afatinib) EGFR tyrosine kinase inhibitors (TKIs) and those with TKI resistance may be given third-generation inhibitor osimertinib. ALK rearrangement cases may be treated with crizotinib. High dose systemic chemotherapy with thiotepa has been tried in these patients with increased risk of hematological toxicity [4]. Intrathecal chemotherapy is commonly offered to these patients, usually through Ommaya reservoir and drugs commonly administered are methotrexate, cytarabine, thiotepa etc. Recently nimotuzumab as targeted agent has been reported to have good tolerance and results, when given via intrathecal route [8].

A high index of suspicion is required to diagnose meningeal carcinomatosis as it largely remains under recognized and overlooked particularly when headache is the sole initial manifestation with no other symptoms of primary lesion elsewhere in the body.

\section{References}

1. Gleissner B, Chamberlain MC. Neoplastic meningitis. Lancet Neurol 2006;5:443-52.

2. Grossman SA, Krabak MJ. Leptomeningeal carcinomatosis. Cancer Treat Rev 1999;25:103-19.

3. Paramez AR, Dixit R, Gupta N, et al. Non-small cell lung carcinoma presenting as carcinomatous meningitis. Lung India 2010;27:158-60.

4. Le Rhun E, Weller M, Brandsma D, et al. EANO-ESMO Clinical Practice Guidelines for diagnosis, treatment and follow-up of patients with leptomeningeal metastasis from solid tumours. Ann Oncol 2017;28:iv84-iv99.

5. Horn VA, Chamberlain MC. Neoplastic meningitis. J Support Oncol 2012;10:45-53.

6. Singh SK, Leeds NE, Ginsberg LE. MR imaging of leptomeningeal metastases: Comparison of three sequences. Am J Neuroradiol 2002;23:817-21.

7. Chamberlain MC. Neoplastic meningitis. Oncologist 2008;13: 967-77.

8. Ju Y, Sun S, Wang J, Jiao S. Prolonged overall survival of patients with leptomeningeal carcinomatosis from non small cell lung cancer. J Can Res Ther 2016;12:126-9. 